\title{
Glutathione Reductase Encoding Gene (gor) is Associated with Oxidative Stress and Antibiotic Susceptibility in Pseudomonas aeruginosa
}

\author{
Ammar Abualnoor ${ }^{a}$ and Dong H. Kwon ${ }^{a, b^{*}}$ \\ ${ }^{a}$ Department of Biology, Long Island University, Brooklyn, New York 11201, USA. \\ ${ }^{b}$ Department of Medicine, Michael E. DeBakey VA Medical Center, Baylor College of Medicine,
} Houston, Texas 77030, USA.

Authors' contributions

This work was carried out in collaboration between both authors. Both authors read and approved the final manuscript.

Article Information

DOI: 10.9734/ARRB/2021/v36i1130444 (1) Dr. Viduranga Y. Waisundara, Australian College of Business \& Technology, Sri Lanka.
Reviewers:

(1) Ionica Mihaela lancu, Banat University of Agronomical Sciences and Veterinary Medicine, Romania. (2) Alfonso Castañeda Martínez, Universidad Autónoma de Nayarit, México. Complete Peer review History: https://www.sdiarticle4.com/review-history/76234

Original Research Article

Received 06 September 2021

Accepted 12 November 2021

Published 18 November 2021

\begin{abstract}
Pseudomonas aeruginosa is a major causative agent of the hospital- and community-acquired infections. These infections are often antibiotic resistant and difficult to treat. Several intrinsic and acquired resistance mechanisms to antibiotics have reported in $P$. aeruginosa. Recently, oxidativestress-scavenging-systems have suggested as a possible intrinsic resistance mechanism to antibiotics because oxidative stresses induced by bactericidal antibiotics contribute to bacterial killing effects. However, this remains controversial such that further clarification is required. Glutathione reductase is a key enzyme in the maintenance of the optimum level of intracellular glutathione-redox potential to ensure normal functioning of cellular processes including the detoxification of oxidative stress. In this study, the role of a glutathione-reductase-encoding gene (gor) in oxidative stress and antibiotic susceptibility was determined in $P$. aeruginosa. Results showed that a gor-mutant strain was more susceptible to hydrogen peroxide (but not superoxide) than the parental strain and $100 \%$ of cells were killed with $0.01 \%$ hydrogen peroxide while the parental strain survived at the same concentration of hydrogen peroxide. The gor-mutant strain was also more susceptible to carbenicillin, chloramphenicol, ciprofloxacin, and tetracycline than the
\end{abstract}


parental strain, which was confirmed by bacterial killing-kinetics. These results suggest that the gor gene is associated with oxidative stress and susceptibility to bactericidal as well as bacteriostatic antibiotics and that the oxidative-stress-scavenging-systems may be a possible drug-target for multidrug resistant $P$. aeruginosa.

Keywords: Glutathione reductase; oxidative stress; antibiotic susceptibility; Pseudomonas aeruginosa.

\section{INTRODUCTION}

Pseudomonas aeruginosa is a Gram-negative human pathogen causing a wide variety of nosocomial and community-acquired infections. The range of $P$. aeruginosa infections varies from localized infections on the human body to lifethreatening systemic disease, including burn wounds, cystic fibrosis, acute leukemia, renal system, bacteremia, urinary tract infection, organ transplants, and intravenous-drug addiction [1, 2]. Treatment of $P$. aeruginosa infections however is difficult due to the presence of antibiotic resistance to a variety of antibiotics, such as aminoglycosides, quinolones, and $\beta$ lactams. The major resistance mechanisms to those antibiotics are intrinsic, acquired, and adaptive resistance. The intrinsic resistance includes decreased-membrane permeability, expression of efflux pumps that expel antibiotics out of the cell and the production of antibioticinactivating enzymes. The acquired resistance can be achieved by either horizontal transfer of resistance genes or alterations on the antibiotic targets. The adaptive resistance involves formation of biofilm in the lungs of infected patients where the biofilm serves as a diffusion barrier to limit antibiotic access to the bacterial cells $[3,4,5]$.

Antibiotics induce oxidative stresses (e.g., hydrogen peroxide, superoxide, and hydroxyl radical) in bacteria. Bactericidal antibiotics (e.g., $\beta$-lactams, aminoglycosides, and quinolones) induce hydroxyl radicals from hydrogen peroxide through the Fenton reaction [6] whereas, in $E$. coli, bacteriostatic antibiotics (e.g., chloramphenicol) induce superoxide [7]. Antibiotic-induced oxidative stresses damage cellular macromolecules and enhance antibiotic lethality (susceptibility) in addition to antibioticspecific killing mechanisms [8]. Therefore, oxidative-stress-scavenging systems consider one of the intrinsic resistant mechanisms to antibiotics.

Cellular metabolism normally produces oxidative stresses in all aerobic organisms. In E. coli, the oxidative stresses activate OxyR and/or SoxRS, which induces the expression of a number of genes including glutathione (GSH) reductase and GSH peroxidase that neutralizes the oxidative stress [9]. For example, hydrogen peroxide is reduced to water and oxygen molecules by GSH peroxidase using electrons from two molecules of $\mathrm{GSH}$, and the two molecules of GSH are oxidized to form GSH disulfide (GSSG). The GSSG is toxic at high levels and reduced back to GSH by the GSH reductase using electrons from NADPH $[9,10]$. In E. coli, the ratio of GSH/GSSG is estimated to be approximately 200 (>99\% of GSH) in growing cells, which corresponds to a redox potential of $-240 \mathrm{mV}$, assuming a total intracellular GSH concentration of $5 \mathrm{mM}, \mathrm{pH} 7.0$, and $25^{\circ} \mathrm{C}$. This $\mathrm{GSH}$-redox system plays a variety of cellular functions not only in detoxifying oxidative stresses but also in deactivation of toxic substances via GSH-conjugate formation [11]. Alterations of the GSH redox potential can impair the functions of GSH-redox system [10]. Therefore, the GSH-redox system is one of the oxidative-stress-scavenging systems and GSH reductase is a key component for this redox system.

These observations suggest that the GSH reductase is associated with oxidative stresses and antibiotic susceptibility. GSH reductase is a dimer composed by two identical subunits with a molecular mass of $55 \mathrm{kD}$, a member of the Flavin-containing enzyme, encoded by a gene (gor) in E. coli [12]. The gor gene from $P$. aeruginosa PAO1 was cloned in E. coli [13], but the roles of the gor gene in oxidative stress and antibiotic susceptibility are currently unclear. In this study, we aimed to understand the role of a glutathione-reductase-encoding gene (gor) in oxidative stress and antibiotic susceptibility. The genes (gor) of $P$. aeruginosa (POA1 and MPAO1) were knocked-out and roles of the gor in oxidative stress and antibiotic susceptibility were determined. The results revealed that the gor-mutant strains were more susceptible to hydrogen peroxide and antibiotics than their parental strains of $P$. aeruginosa, suggesting the gor gene may be associated with susceptibility of antibiotics and the gor gene may be a possible 
drug-target for the antibiotic resistant $P$. aeruginosa.

\section{MATERIALS AND METHODS}

\subsection{Bacterial Strains, Growth Conditions, and Chemicals}

$P$. aeruginosa PAO1 was obtained from the previous studies [14]. P. aeruginosa MPAO1 and a gor-mutant strain (PW4508; gor::TnTc) were obtained from the sequence-verified transposon mutant library (University of Washington, Seattle, WA). The bacterial strains routinely grew on Luria-Bertani (LB; Becton, Dickinson and Company, Sparks, MD) agar plates or broth at $37^{\circ} \mathrm{C}$. The mutant strain grew in a minimal medium for $P$. aeruginosa containing glutamate as a sole carbon and nitrogen source as described [14]. All antibiotics, Lglutathione (reduced-glutathione), and other chemicals were purchased from Sigma-Aldrich (St. Louis, MO).

\subsection{Cloning and Gene-knockout}

Genomic DNA extracted from $P$. aeruginosa PAO1 was used to amplify a full length of a glutathione reductase encoding gene (gor) encompassing upstream (570-bp) and downstream (60-bp) of the gor gene (1980-bp: forward PCR-primer: 5'-cagggaatcggcagcgcct-3'; reverse PCR- primer: 5'-tgcgcgcgaaaatgaaagaa$\left.3^{\prime}\right)$. The PCR fragment was inserted into an $E$. colil $P$. aeruginosa shuttle vector (named pAU250) and used to determine DNA sequence from the commercial service (GENEWIZ, South Plainfield, NJ). The gene-knockout experiment was performed as described previously [14]. Briefly, the cloned gor gene was inactivated by inserting a gentamicin-resistance gene cassette $(\mathrm{Gm})$ from $\mathrm{pGM} \Omega 1$ into a middle of the gene (Eco47III) and the knocked-out gene cassette (gor::Gm) was transferred into a suicidal conjugative vector (pRTP1). E. coli SM10 carrying the resulting plasmid was used as a donor strain and $P$. aeruginosa PAO1 was used for the recipient strain. Bi-parental conjugation was performed as described [14] and the genereplaced $P$. aeruginosa (gor::Gm) was selected on LB agar plates containing gentamicin (80 $\mu \mathrm{g} / \mathrm{ml}$ ) for $P$. aeruginosa and chloramphenicol $(15 \mu \mathrm{g} / \mathrm{ml})$ for counter-selection against $E$. coli SM10. Authenticity of the gene-knockout was confirmed by PCR amplication of the upstream and downstream of the gor gene.

\subsection{Glutathione Reductase Enzyme Assay}

Overnight-cultured cells were diluted (1/100) into fresh LB broth $(30 \mathrm{ml})$ and grown on a rotary shaker $(250 \mathrm{rpm})$ at $37^{\circ} \mathrm{C}$. The cells $(5 \mathrm{ml})$ at optical density $\left(\mathrm{OD}_{600}\right)$ of 1.0 were washed three times with phosphate buffer $(\mathrm{pH} 7.0)$ and disrupted by sonication as described [15]. The crude extract of the cells spun down at $15,000 \mathrm{rpm}$ for $5 \mathrm{~min}$ and the supernatant used to measure levels of GSH reductase. The protein concentration of the crude extract was measured using the Coomassie PlusTM Protein Assay Reagent (Thermo Scientific, Rockford, IL). The amount of GSH reductase (units/mg protein) was determined using a commercial kit (SigmaAldrich, St. Louis, MO) as suggested by the vendor.

\subsection{Determination of Minimum Inhibitory Concentration (MIC) of Hydrogen Peroxide, Paraquat, and Antibiotics}

MIC levels of hydrogen peroxide, paraquat, and antibiotics were determined as guided by the Clinical and Laboratory Standards Institute (CLSI) as described previously [14] with a minor modification. Briefly, two-fold serial dilutions of hydrogen peroxide, paraquat, and antibiotics were performed using Mueller-Hinton broth (MHB; Oxoid, Ogdensburg, NY) and fresh overnight cultures of $P$. aeruginosa strains $\left(\sim 10^{8}\right.$ viable cells per $\mathrm{ml}$ ) inoculated at each of the dilutions. The cells were incubated overnight without shaking at $37^{\circ} \mathrm{C}$. MIC levels defined as the lowest concentration of hydrogen peroxide, paraquat, and antibiotics that completely inhibited cellular growth of the inoculum. Determination of MIC levels was repeated three times to confirm the results.

\subsection{Bacterial Killing Assay}

Bacterial killing of $P$. aeruginosa strains were determined as previously described [16] with a minor modification. Briefly, hydrogen peroxide or antibiotics at different concentrations were added in $\mathrm{MH}$ broth (1 $\mathrm{ml}$ in the Falcon culture tubes) and fresh cultures of cells $\left(\sim 10^{8}\right.$ viable cells per $\mathrm{ml}$ ) were inoculated into each of the concentrations. The cells were incubated at $37^{\circ} \mathrm{C}$ without shaking for 18 hours and spread on plain LB agar plates with appropriate dilutions. The LB agar plates were incubated for 24 hours at $37^{\circ} \mathrm{C}$ and colony-forming units (CFU) were counted 
per $\mathrm{ml}$. The bacterial killing assay repeated three times to confirm the results.

\section{RESULTS}

\subsection{Mutant Strains of $P$. aeruginosa}

A mutant strain of $P$. aeruginosa PAO1 (gor::Gm) was constructed as described in the Materials and Methods. The mutant strain of $P$. aeruginosa MPAO1 (gor::TnTc) obtained from the mutant library (University of Washington, Seattle, WA) was confirmed by PCR-sequencing as suggested by the mutant library. The two mutant strains were used to determine GSH reductase activity with comparison to their parental strains. Results showed that the GSH reductase activity (units $/ \mathrm{mg}$ protein) of the mutant strains was significantly lower than that of their parental strains $[2.25 \pm 0.14$ for PAO1 and $1.69 \pm 0.25$ for MPAO1; $0.18 \pm 0.03$ for PAO1 (gor::Gm) and $0.12 \pm 0.02$ for MPAO1 (gor::TnTc)]. The growth rate of the gor-mutant strains was similar to their parental strains in the minimum medium (data not shown).

(A)

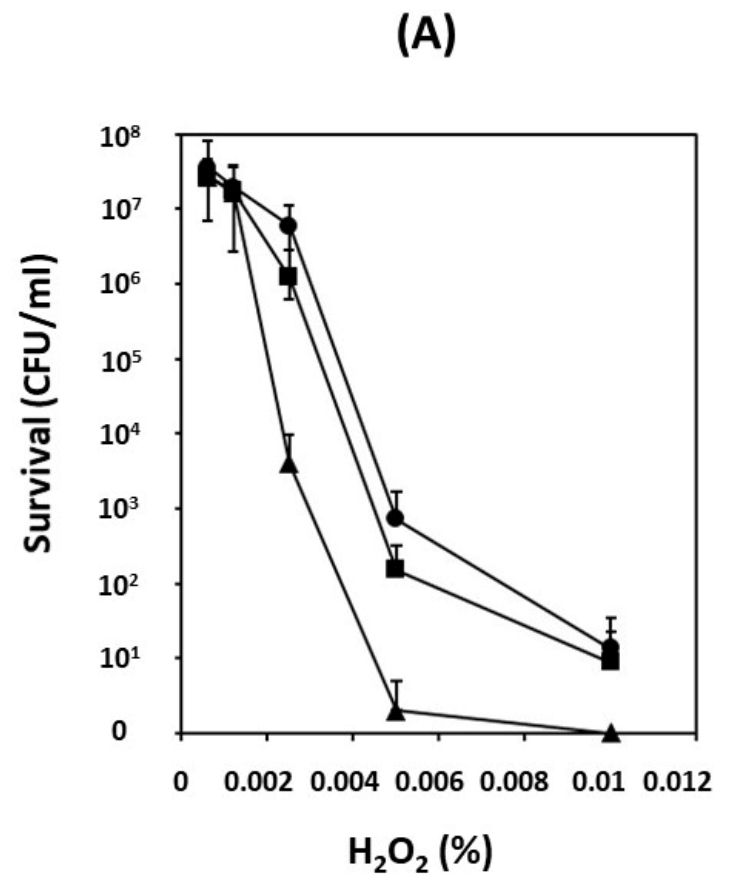

\subsection{Effect of a GSH Reductase-encoding Gene (gor) on Oxidative Stresses}

The two gor-mutant strains and their parental strains were used to determine levels of MIC against hydrogen peroxide and superoxideproducing paraquat. Results showed that MIC levels of the gor-mutant strains against hydrogen peroxide were 2-fold lower than that of their parental strains (MICs fall from 0.01 to $0.005 \%$ ). The levels of MIC were fully restored in the mutant strains harboring a plasmid carrying an intact gor gene. MIC levels of the mutant and their parental strains against paraquat were all $200 \mu \mathrm{g} / \mathrm{ml}$ (Table 1). To clarify the effects of gor on hydrogen peroxide, bacterial killing assay was determined at different concentrations of hydrogen peroxide. As shown in Fig. 1, both gormutant strains were completely killed at $0.01 \%$ hydrogen peroxide while their parental strains survived significantly at the same concentration of hydrogen peroxide. Survival of the mutant strains harboring a plasmid carrying an intact gor gene was similar as their parental strains (Fig. 1). These results suggest that the GSH reductaseencoding gene (gor) is associated with oxidative stress in $P$. aeruginosa.

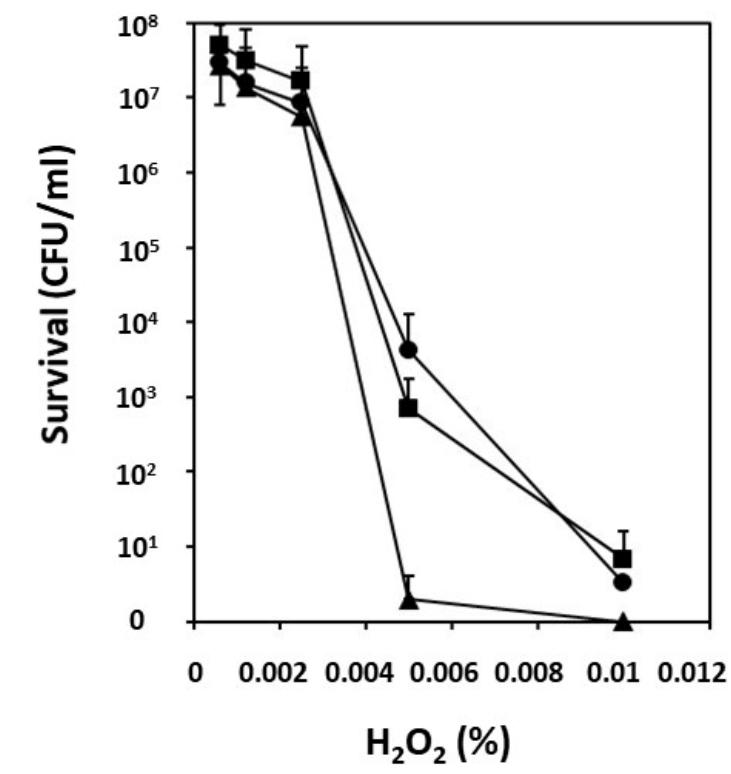

Fig. 1. Bacterial killing of hydrogen peroxide $\left(\mathrm{H}_{2} \mathrm{O}_{2}\right)$ on $P$. aeruginosa

The bacterial killing assay performed as described in Materials and Methods. $(A)$ is $P$. aeruginosa $P A O 1$ and $(B)$ is $P$. aeruginosa MPAO1. For $(A)$ and $(B)$, circle is wild type strains, triangle is mutant strains (gor::Gm for PAO1 and gor::TnTc for MPAO1), and square is gor-mutant stains carrying an intact gor gene (pAU250). Three independent measurements used for the standard deviation 
Table 1. Hydrogen peroxide and paraquat susceptibility in $P$. aeruginosa

\begin{tabular}{lll}
\hline Strain & \multicolumn{2}{c}{ Minimum Inhibitory Concentration $(\mathbf{M I C})^{\mathbf{a}}$} \\
\cline { 2 - 3 } & Hydrogen peroxide $\left(\mathbf{H}_{2} \mathbf{O}_{2} ; \%\right)$ & Paraquat $(\boldsymbol{\mu g} / \mathbf{m l})$ \\
\hline PAO1 & 0.01 & 200 \\
PAO1 (gor::Gm) & 0.005 & 200 \\
PAO1 (gor::Gm/pAU250) ${ }^{\mathrm{b}}$ & 0.01 & 200 \\
MPAO1 & 0.01 & 200 \\
MPAO1 (gor::TnTc) & 0.005 & 200 \\
MPAO1 (gor::TnTc/pAU250) & 0.02 & 200 \\
\hline \multicolumn{3}{c}{${ }^{2}$ MIC measurement repeated three times with an identical result. } \\
\end{tabular}

(A)
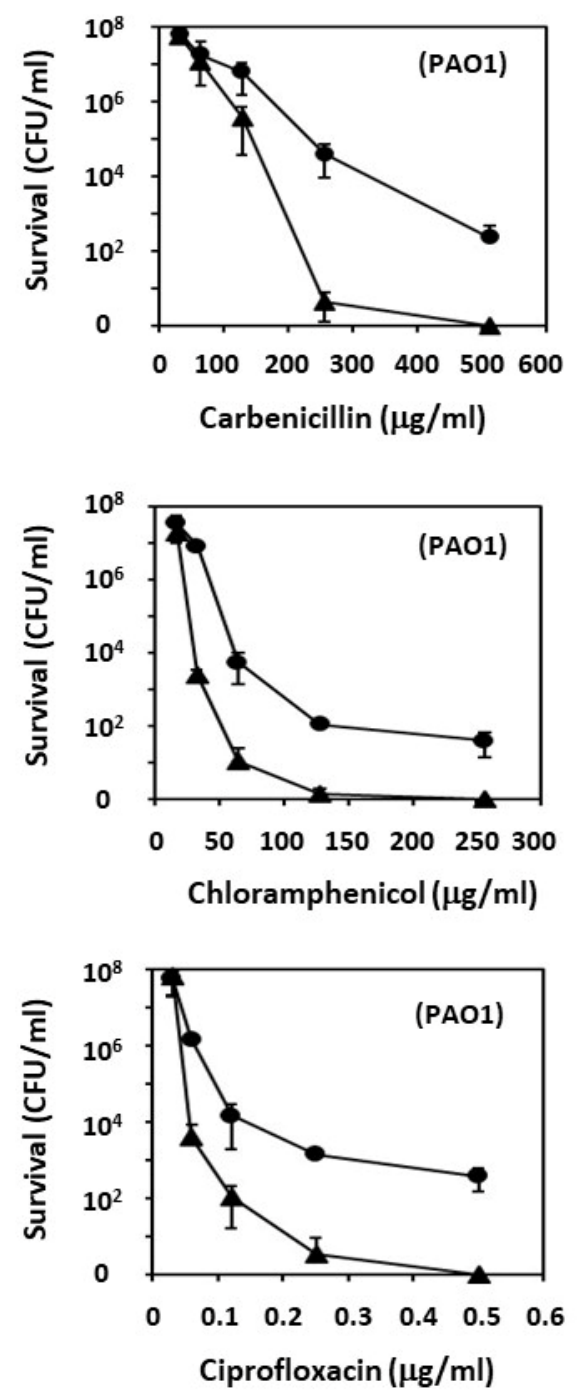

(B)
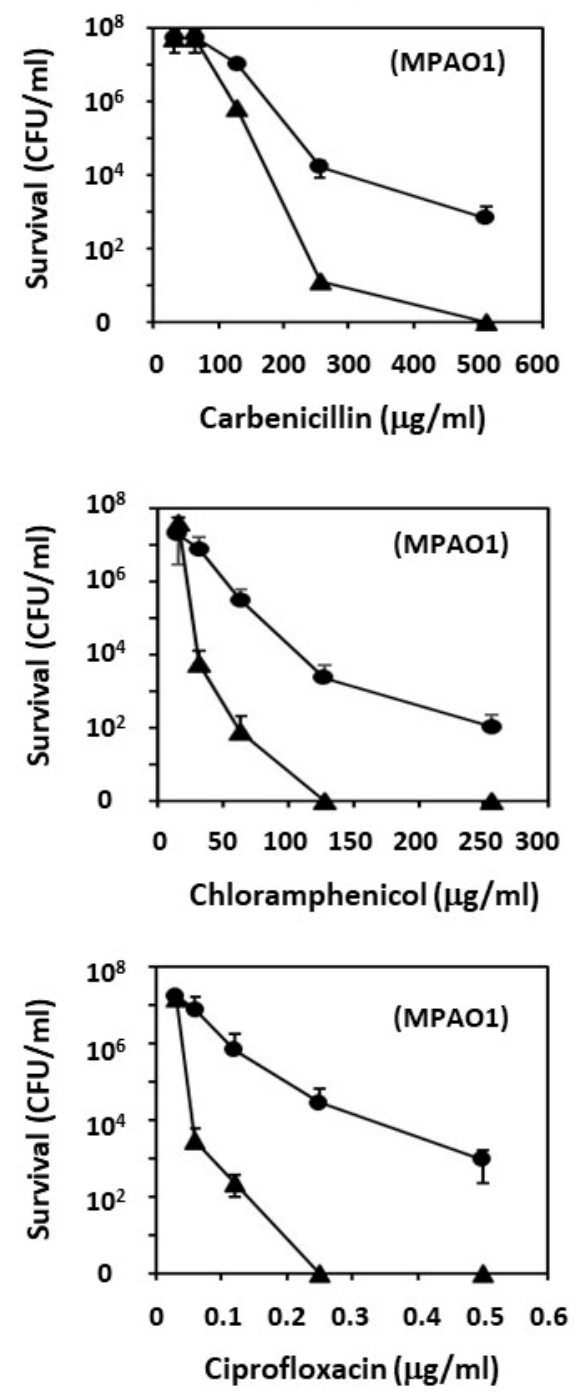

Fig. 2. Bacterial killing of antibiotics on $P$. aeruginosa

The bacterial killing assay performed as described in Materials and Methods. $(A)$ is P. aeruginosa PAO1 and (B) is $P$. aeruginosa MPAO1. For $(A)$ and $(B)$, circle is wild type strains and triangle is mutant strains (gor::Gm for PAO1 and gor::TnTC for MPAO1). Three independent measurements used for the standard deviation 
Table 2. Antibiotic susceptibility in P. aeruginosa

\begin{tabular}{lllllllll}
\hline & \multicolumn{8}{c}{ Minimum Inhibitory Concentration (MIC; $\boldsymbol{\mu g} / \mathbf{m l})^{\text {a }}$} \\
\cline { 2 - 9 } & ATM & CAR & CAZ & MEM & CHL & CIP & GEN & TET \\
\hline PAO1 & 4 & 128 & 4 & 2 & 64 & 0.12 & ND & 8 \\
PAO1 (gor::Gm) & 4 & 64 & 4 & 2 & 32 & 0.062 & ND & 4 \\
MPAO1 & 2 & 256 & 4 & 1 & 128 & 0.25 & 0.5 & ND \\
MPAO1 (gor::TnTc) & 2 & 128 & 4 & 1 & 32 & 0.062 & 0.25 & ND \\
\hline \multicolumn{7}{l}{} \\
\hline
\end{tabular}

Note: ATM: aztreonam; CAR: carbenicillin; CAZ: ceftazidime; MEM: meropenem; CHL: chloramphenicol; CIP: ciprofloxacin; GEN: gentamicin; TET: tetracycline ND: not determined

\subsection{Role of a GSH Reductase-encoding Gene (gor) in Antibiotic Susceptibility}

Antibiotics induce oxidative stresses that contribute bacterial killing effect [8]. Since the gor gene is associated with oxidative stress as shown by the above results, the gor gene may be also associated with antibiotic susceptibility. To test this possibility, the gor-mutant strains were used to determine antibiotic susceptibility in comparison to their parental strains. Results revealed that the mutant strains were both more susceptible to carbenicillin, chloramphenicol, ciprofloxacin, and tetracycline than their parental strains (MICs fall 2- to 4-fold) (Table 2). To corroborate these results bacterial killing assay was determined at different concentrations of antibiotics. As shown in Fig. 2, the gor-mutant strains were completely killed at much lower concentrations of each antibiotic (carbenicillin, chloramphenicol, and ciprofloxacin) than their parental strains. These results are consistent with the MIC results (Table 2) and suggest that the gor gene is also associated with antibiotic susceptibility in $P$. aeruginosa.

\section{DISCUSSION}

$P$. aeruginosa is a common Gram-negative rodshaped bacterium associated with a variety of infections in hospitalized and immunocompromised people. Infections with $P$. aeruginosa can lead to severe illness and death. The CDC (Centers for Disease Control and Prevention) reported 51,000 health-care-associated $P$. aeruginosa infections per year and 440 of them were lethal. These 440 deaths were among 6,700 infections with multidrug resistant $P$. aeruginosa (https://www.cdc.gov/hai/organisms/pseudomona s.html). $P$. aeruginosa, unlike other Gramnegative bacterial pathogens, constitutively expresses chromosomal-encoded genes for AmpC $\beta$-lactamase and multidrug efflux pumps and has a low permeability outer membrane, which produces high-level intrinsic resistance to diverse antibiotics. Additionally, $P$. aeruginosa can acquire resistance to most commercially available antibiotics [17, 3]. Recently, oxidativestress-scavenging-system (OSSS) has suggested as one of the intrinsic resistant mechanisms to antibiotics based on the evidence that OSSS detoxifies oxidative stresses induced by antibiotics. This hypothesis however requires further clarification [8].

Three major OSSS exist in bacteria such as an enzymatic system (e.g., catalases and superoxide dismutases), a GSH-redox system, and a thioredoxin redox system [18]. In this study, we determined roles of a gor gene encoding GSH reductase in oxidative stress and antibiotic susceptibility. GSH reductase activity was significantly decreased in the gor-mutant strains but was not fully deficient, suggesting an alternative pathway (or enzyme) to salvage the role of GSH-redox system. Similar growth rates of the gor-mutant and the parental strains also support this possibility. GSH reductase is a key component of the GSH-redox system that plays a key role in maintaining an optimum level of the intracellular redox potential and is required for normal cellular processes in E. coli [10]. In eukaryotic cells, GSH-redox and thioredoxin redox systems crosstalk to compensate roles for each other [18] and this may also be the case in $P$. aeruginosa.

MIC levels of the gor-mutant strains against hydrogen peroxide were lower than their parental strains. In addition, killing-rate of the gor-mutant strains was faster than their parental strains at the same concentrations of hydrogen peroxide. These results suggest that the lack of GSH reductase (gor-mutation) is associated with susceptibility to hydrogen peroxide. This may be due to an unbalance of the ratio of GSH/GSSG, which directly depends on the GSH reductase and relates to the function of GSH peroxidase 
that detoxifies hydrogen peroxide to water and oxygen molecules. Superoxide susceptibility of the gor-mutant strains was the same (or similar) level as their parental strains, suggesting that GSH reductase may not be responsible for detoxifying superoxide. Superoxide dismutase normally detoxifies the superoxide in bacteria, and $P$. aeruginosa encodes superoxide dismutase (http://www.pseudomonas.com/).

Kohanski et al. and other investigators reported that hydroxyl radicals were induced by bactericidal antibiotics (e.g., ampicillin, kanamycin, norfloxacin) but not by bacteriostatic antibiotics (e.g., chloramphenicol, rifampicin, and tetracycline), which enhanced the bacterial killing effect [6]. We showed that the gor gene was associated with oxidative stress, thus, the gormutant strains should be more susceptible to the bactericidal antibiotics than their parental strains. We observed that the gor-mutant strains were more susceptible to bactericidal antibiotics (carbenicillin and ciprofloxacin) than their parental strain, which is consistent with the report from Kohanski et al. However, our results showed that the gor-mutant strains were also more susceptible to bacteriostatic antibiotics (chloramphenicol and tetracycline) than their parental strain. These results may be related to the fact that the gor gene controls the GSH-redox system that detoxifies a broad range of toxic substances.

\section{CONCLUSION}

Overall, this study demonstrates that the gor gene is associated with oxidative stress as well as antibiotic susceptibility in $P$. aeruginosa. The gor gene is a key component of the GSH-redox system and thus the GSH-redox system may be a possible drug target to treat the multidrug resistant $P$. aeruginosa.

\section{ACKNOWLEDGEMENTS}

We thank Dr. David Y. Graham for carful reviewing this manuscript. This study was partially supported by graduate student funds from Biology department (Long Island University, Brooklyn, NY)

\section{COMPETING INTERESTS}

Authors have declared that no competing interests exist.

\section{REFERENCES}

1. Richards MJ, Edwards JR, Culver DH, Gaynes RP. Nosocomial infections in pediatric intensive care units in the United States. National Nosocomial Infections Surveillance System. Pediatrics. 1999;103:e39.

2. Matta $R$, Hallit $S$, Hallit $R$, Bawab $W$, Rogues AM, Salameh P. Epidemiology and microbiological profile comparison between community and hospital acquired infections: A multicenter retrospective study in Lebanon. Journal of Infection and Public Health. 2018;11:405-411.

3. Zavascki AP, Carvalhaes CG, Picao RC, Gales AC. Multidrug-resistant Pseudomonas aeruginosa and Acinetobacter baumannii: resistance mechanisms and implications for therapy. Expert Review of Anti-infective Therapy. 2010;8:71-93.

4. Cerceo E, Deitelzweig SB, Sherman BM, Amin AN. Multidrug-resistant gramnegative bacterial infections in the hospital setting: Overview, implications for clinical practice, and emerging treatment options. Microb Drug Resist. 2016;22:412-431.

5. Pang Z, Raudonis R, Glick BR, Lin TJ, Cheng $Z$. Antibiotic resistance in Pseudomonas aeruginosa: mechanisms and alternative therapeutic strategies. Biotechnol Adv. 2019;37:177-192.

6. Kohanski MA, Dwyer DJ, Hayete B, Lawrence CA, Collins JJ. A common mechanism of cellular death induced by bactericidal antibiotics. Cell. 2007;130:797810.

7. Albesa I, Becerra MC, Battan PC, Paez $\mathrm{PL}$. Oxidative stress involved in the antibacterial action of different antibiotics. Biochemical and Biophysical Research Communications. 2004;317:605- 609.

8. Van Acker $\mathrm{H}$, Coenye $\mathrm{T}$. The Role of Reactive Oxygen Species in AntibioticMediated Killing of Bacteria. Trends in Microbiology. 2017;25:456-466.

9. Green J, Paget MS. Bacterial redox sensors. Nature reviews Microbiology. 2004;2:954-966.

10. Smirnova GV, Oktyabrsky ON. Glutathione in bacteria. Biochemistry Biokhimiia. 2005;70:1199-1211.

11. Couto N, Wood J, Barber J. The role of glutathione reductase and related enzymes on cellular redox homoeostasis network. 
Free Radical Biology \& Medicine. 2016;95:27-42.

12. Jiang F, Hellman U, Sroga GE, Bergman $B$, Mannervik B. Cloning, sequencing, and regulation of the glutathione reductase gene from the cyanobacterium Anabaena PCC 7120. The Journal of Biological Chemistry. 1995;270:22882-22889.

13. Perr ACF, Bhriain NN, Brown NL, Rouch DA. Molecular characterization of the gor gene encoding glutathione reductase from Pseudomonas aeruginosa: determinants of substrate specificity among pyridine nucleotide-disulphide oxidoreductases. Molecular Microbiology. 1991;5:163-171.

14. Kwon DH, Lu CD. Polyamines induce resistance to cationic peptide, aminoglycoside, and quinolone antibiotics in Pseudomonas aeruginosa PAO1. Antimicrobial Agents and Chemotherapy. 2006;50:1615-1622.

15. Kwon DH, Hekmaty S, Seecoomar G. Homeostasis of glutathione is associated with polyamine-mediated beta-lactam susceptibility in Acinetobacter baumannii ATCC 19606. Antimicrob Agents Chemother. 2013;57:5457-5461.

16. Kanagaratnam $R$, Sheikh $R$, Alharbi $F$, Kwon DH. An efflux pump (MexAB-OprM) of Pseudomonas aeruginosa is associated with antibacterial activity of Epigallocatechin-3-gallate (EGCG). Phytomedicine. 2017;36:194-200.

17. Falagas ME, Rafailidis PI, Matthaiou DK, Virtzili S, Nikita D, Michalopoulos A. Pandrug- resistant Klebsiella pneumoniae, Pseudomonas aeruginosa and Acinetobacter baumannii infections: characteristics and outcome in a series of 28 patients. International Journal of Antimicrobial Agents. 2008;32:450454.

18. Lu J, Holmgren A. The thioredoxin antioxidant system. Free Radic Biol Med. 2014;66:75-87.

(0) 2021 Abualnoor and Kwon; This is an Open Access article distributed under the terms of the Creative Commons Attribution License (http://creativecommons.org/licenses/by/4.0), which permits unrestricted use, distribution, and reproduction in any medium, provided the original work is properly cited.

The peer review history for this paper can be accessed here: https://www.sdiarticle4.com/review-history/76234 